See discussions, stats, and author profiles for this publication at: https://www.researchgate.net/publication/330396507

\title{
Was Einstein in need to impose the stability of the speed of light in the Theory of Special Relativity
}

Research · January 2019

DOI: $10.13140 /$ RG.2.2.16606.28485

CITATIONS

READS

0

251

1 author:

Mohamed AHMED Abouzeid

luxor stem school

15 PUBLICATIONS O CITATIONS

SEE PROFILE

Some of the authors of this publication are also working on these related projects:

Project Was Einstein in need to impose the stability of the speed of light in the Theory of Special Relativity View project

Project Theory of constants View project 
International Journal of Scientific \& Engineering Research Volume 9, Issue 12, December-2018

Was Einstein in need to impose the stability of the speed of light in

\section{the Theory of Special Relativity?}

According to Einstein's first hypothesis only, it can be reached to transfer formats between reference frames in the special theory of relativity

Consider two observers $A, B$ in both frames $S_{1}, S_{2}$

At first $T_{1}=T_{2}=0$

: $T_{1}$ The time at frame $S_{1}$ and $T_{2}$ the time at frame $S_{2}$

Let $A$ and $B$ in the same place and at the same time, they each send a light signal

Let $S_{2}$ and (observer $\mathrm{B}$ ) moving respect to $S_{1}$ and (observer A) with uniform Velocity $\vec{V}$

At the direction of the axis $\overrightarrow{o x}$

In this case the signal is spread as a spherical wave

\section{Measurements A:}

At the moment $T_{1}$ of his watch, the wave equation appears in the Formula:

$X_{1}{ }^{2}+Y_{1}{ }^{2}+Z_{1}{ }^{2}-C_{1}{ }^{2} T_{1}{ }^{2}=0$

$C_{1}$ Speed of light at fame $S_{1}$

\section{Measurements B:}

At moment $T_{2}$ of his watch, the wave equation appears in the Formula:

$X_{2}{ }^{2}+Y_{2}{ }^{2}+Z_{2}{ }^{2}-C_{2}{ }^{2} T_{2}{ }^{2}=0$

$C_{2}$ Speed of light at fame $S_{2}$

Notice $C_{1} \neq C_{2}$ 
Then

$X_{1}{ }^{2}+Y_{1}{ }^{2}+Z_{1}{ }^{2}-C_{1}{ }^{2} T_{1}{ }^{2}=X_{2}{ }^{2}+Y_{2}{ }^{2}+Z_{2}{ }^{2}-C_{2}{ }^{2} T_{2}{ }^{2}$

Let $Y_{1}=Y_{2}$ and $Z_{1}=Z_{2}$

So

$X_{1}{ }^{2}-C_{1}{ }^{2} T_{1}{ }^{2}=X_{2}{ }^{2}-C_{2}{ }^{2} T_{2}{ }^{2}$

Let

$X_{2}=G_{11} X_{1}+G_{14} T_{1}$

$T_{2}=G_{41} X_{1}+G_{44} T_{1}$

Where

$G_{11}, G_{14}, G_{41}, G_{44}$ are constants

Consider the moving of origin point $O_{2}$ respect to $S_{1}$

(Ordinates $\mathrm{O}_{2}$ ) is $X_{2}=0$

So from equation (5)

$0=G_{11} X_{1}+G_{14} T_{1}$

$G_{14} T_{1}=-G_{11} X_{1}$

$G_{14}=-G_{11} \frac{X_{1}}{T_{1}} \quad$ And $\frac{X_{1}}{T_{1}}=V$

So $G_{14}=-G_{11} V$ 
(Ordinates $O_{1}$ ) is $X_{1}=0$

So from equation (5)

$X_{2}=0+G_{14} T_{1}$

$X_{2}=G_{14} T_{1}$

From equation (6)

$T_{2}=G_{41} X_{1}+G_{44} T_{1}$

$T_{2}=0+G_{44} T_{1}$

$T_{2}=G_{44} T_{1}$

From equation (8) and (9)

$\frac{X_{2}}{T_{2}}=\frac{G_{14} T_{1}}{G_{44} T_{1}} \quad$ And $\quad \frac{X_{2}}{T_{2}}=-V$

So $\frac{G_{14}}{G_{44}}=-V$

From equation (7)

$\frac{-G_{11} V}{G_{44}}=-V$

$G_{44}=G_{11}$

From equations (5), (7)

$X_{2}=G_{11} X_{1}-G_{11} V T_{1}$

So

$X_{2}=G_{11}\left(X_{1}-V T_{1}\right)$

From equations (6), (10)

$T_{2}=G_{41} X_{1}+G_{11} T_{1}$

From equations (4), (11), (12)

$X_{1}{ }^{2}-C_{1}{ }^{2} T_{1}{ }^{2}=X_{2}{ }^{2}-C_{2}{ }^{2} T_{2}{ }^{2}$

$X_{1}{ }^{2}-C_{1}{ }^{2} T_{1}{ }^{2}=G_{11}{ }^{2}\left(X_{1}-V T_{1}\right)^{2}-C_{2}{ }^{2}\left(G_{41} X_{1}+G_{11} T_{1}\right)^{2}$ 
Compare the coefficient of $X_{1}{ }^{2}$

$1=G_{11}{ }^{2}-C_{2}{ }^{2}\left(G_{41}\right)^{2}$

$G_{11}{ }^{2}=1+C_{2}{ }^{2}\left(G_{41}\right)^{2}$

Compare the coefficient of $X_{1} T_{1}$

$0=-2 V G_{11}{ }^{2}-2 C_{2}{ }^{2}\left(G_{41} G_{11}\right)$

$0=V G_{11}{ }^{2}+C_{2}{ }^{2}\left(G_{41} G_{11}\right)$

$V G_{11}{ }^{2}=-C_{2}{ }^{2}\left(G_{41} G_{11}\right)$

$V G_{11}=-C_{2}^{2}\left(G_{41}\right)$

$G_{11}=\frac{-C_{2}^{2}}{V}\left(G_{41}\right)$

$G_{41}=\frac{-V}{C_{2}^{2}}\left(G_{11}\right)$

From equation (15) in (12)

$T_{2}=\frac{-V}{C_{2}{ }^{2}}\left(G_{11}\right) X_{1}+G_{11} T_{1}$

$T_{2}=G_{11}\left(T_{1}-\frac{V}{C_{2}{ }^{2}} X_{1}\right)$

From equation (14), (15)

$G_{11}^{2}=1+C_{2}^{2}\left(\frac{-V}{C_{2}^{2}}\left(G_{11}\right)\right)^{2}$ 
$G_{11}^{2}=1+\frac{V^{2}}{C_{2}{ }^{2}}\left(G_{11}\right)^{2}$

$G_{11}^{2}-\frac{V^{2}}{{C_{2}}^{2}}\left(G_{11}\right)^{2}=1$

$G_{11}^{2}\left(1-\frac{V^{2}}{C_{2}{ }^{2}}\right)=1$

$G_{11}{ }^{2}=\frac{1}{\left(1-\frac{V^{2}}{C_{2}{ }^{2}}\right)}$

Compare the coefficient of $T_{1}{ }^{2}$

$-C_{1}{ }^{2}=G_{11}{ }^{2}(-V)^{2}-C_{2}{ }^{2}\left(G_{11}\right)^{2}$

$-C_{1}{ }^{2}=G_{11}{ }^{2}\left(V^{2}-C_{2}{ }^{2}\right)$

$G_{11}{ }^{2}=\frac{-C_{1}{ }^{2}}{\left(V^{2}-C_{2}{ }^{2}\right)}$

Equation $17=18$

$\frac{-C_{1}{ }^{2}}{\left(V^{2}-C_{2}{ }^{2}\right)}=\frac{1}{\left(1-\frac{V^{2}}{C_{2}{ }^{2}}\right)}$

$$
\left(V^{2}-C_{2}{ }^{2}\right)=-C_{1}{ }^{2}\left(1-\frac{V^{2}}{C_{2}{ }^{2}}\right)
$$

$\left(\frac{C_{2}^{2}}{C_{1}{ }^{2}}-\frac{V^{2}}{C_{1}{ }^{2}}\right)=\left(1-\frac{V^{2}}{C_{2}{ }^{2}}\right)$

Compare the coefficient

Then $C_{1}{ }^{2}=C_{2}{ }^{2}$

So 
$C_{1}= \pm C_{2}$

\section{Einstein was not to need to impose the stability of the speed of light in the Theory of Special Relativity}

Then

$G_{11}{ }^{2}=\frac{-C_{1}{ }^{2}}{\left(V^{2}-C_{1}{ }^{2}\right)}$

$G_{11}=\sqrt{\frac{-C_{1}{ }^{2}}{\left(V^{2}-C_{1}{ }^{2}\right)}}$

$G_{11}=\sqrt{\frac{1}{\left.1-\frac{\left(V^{2}\right.}{C_{1}{ }^{2}}\right)}}$

So transformation is

$X_{2}=G_{11}\left(X_{1}-V T_{1}\right)$

$T_{2}=G_{11}\left(T_{1}-\frac{V}{C_{2}{ }^{2}} X_{1}\right)$

$G_{11}=\sqrt{\frac{1}{\left.1-\frac{\left(V^{2}\right.}{C_{1}{ }^{2}}\right)}}$ 